\title{
Self-trained perception need not be veridical: striking can exaggerate judgment by wielding and can transfer exaggeration to new stimuli
}

\author{
Damian G. Kelty-Stephen ${ }^{1}$ - Charles L. Eddy ${ }^{1}$
}

Published online: 17 June 2015

(C) The Psychonomic Society, Inc. 2015

\begin{abstract}
Previous literature on self-training dynamic touch suggested that haptic judgments of length following wielding might benefit from new information through participants' own striking actions with the same stimuli. However, the conclusion that this self-training tended towards a veridical outcome of zero discrepancy between actual length and judged length was premature. In this replication, we allowed adult participants $(n=15)$ to strike on each trial and changed the stimuli in mid-experiment to determine whether striking helped participants build more accurate perceptions of length transferrable from one stimulus scale to another. We predicted that, if selftraining led to better length judgments, the repeated striking would improve judgments and that, in turn, judgments following the switch of stimuli would show a good transfer of what participants had learned. On the other hand, self-training may simply exaggerate inertial properties of stimuli and may be sensitive to sudden changes in the scale of stimuli. Mixedeffect modeling of discrepancies show that striking only accentuated effects of inertial moment, producing exaggerated length judgments. Correlation between perceived length and actual length increased only for participants who experienced a switch in individual stimuli but not stimulus scale. We discuss the implications of these findings for any theoretical relationship between self-organization and veridicality.
\end{abstract}

Keywords Haptics $\cdot$ Perceptual learning $\cdot$ Perception and action

Damian G. Kelty-Stephen

keltysda@grinnell.edu

1 Grinnell College, 1115 8th Ave., Grinnell, IA 50112, USA

\section{Introduction}

Prior work tested whether wielding and striking unseen objects would inform judgments of the objects' length (Stephen \& Arzamarski, 2009). This work found that participants generally underestimated the veridical length, with discrepancies between judged and actual length following a negative linear relationship with the logarithm of the first moment of inertia $\left(\log I_{1}\right) .{ }^{1}$ Striking appeared to improve judgments for those objects with greater $\log I_{1}$. However, did that increase in judgment reflect more accuracy or just overestimation?

To test between these possibilities, we used a stimulus set of roughly homogeneous wooden dowels, and tested participants' dynamic-touch length judgments for non-overlapping sets of dowels over each of three blocks. ${ }^{2}$ To accentuate any positive effects of self-training, we allowed participants to strike an unseen surface for mechanical feedback on every

\footnotetext{
${ }^{1}$ Previous research showed that perceived length in dynamic touch may be a power function of the first moment of inertia - a mechanical quantity related to the square of length (Fitzpatrick, Carello, \& Turvey, 1994). Regression modeling of the logarithm of perceived length might thus estimate the power-function exponents as the coefficients for logarithm of the first moment of inertia. However, perceived length may also be a more straightforward function of the length to center of mass, a quantity related to length and not length-squared (van de Langenberg, Kingma, \& Beek, 2008). Whether or not perceived length is a power function of inertial moments, logarithmic scaling has the benefit of minimizing leverage effects from extremal values of inertial as it increases nonlinearly while stimulus sets (e.g., the present one) might be designed to have linear increase in length.

${ }^{2}$ Rods were wooden dowels purchased at a local hardware store. It is reasonable to assume homogeneous density within each rod, but, because the rods were natural materials, densities varied somewhat across dowels. Nevertheless, even with that natural variation, $\operatorname{logI1}$ values were highly correlated with log rod length $(r>.997)$.
} 
single trial. We might expect a roughly cubic trajectory of discrepancy between judged and actual length with discrepancies dropping to zero after initial transients of incorrect guesses and overcorrection. To begin with, we expected underestimation at the beginning (e.g., Stephen \& Arzamarski, 2009), and then we expected regression to the mean (i.e., towards zero-discrepancy) as participants took advantage of striking the unseen surface. At a certain point, participants might overcorrect, past zero-discrepancy and into positive discrepancy, and then temper their overcorrection by settling back towards zero-discrepancy. If self-training dynamic touch makes length judgments more accurate, switching the scale of the stimulus objects might introduce another transient of inaccuracy. As participants reorient to what will obviously feel different, we should expect discrepancy to diminish to zero according to a similarly cubic pattern as above (Fig. 1).

Crucially, if striking solidifies more veridical understanding of length (i.e., closer to zero discrepancy), this understanding should not only stabilize towards zerodiscrepancy but also transfer to new stimuli. Two groups used either a Short stimulus subset followed by a Long subset or vice versa (henceforth, we label conditions Short-Long or Long-Short, respectively). A third Interleaved group received the full range of stimulus set throughout, including every other available length and so spanning the entire set's range of $\log I_{1}$, switching only from odd-numbered stimuli to even-numbered stimuli. Given the full range of stimuli throughout, this third group should show discrepancies converging to zero without interruption through the third block.

If self-training disposes the haptic perceptual system to overestimate the length of stimulus objects with greater $\log \mathrm{I}_{1}$, we should find this positive interaction of striking with $\log \mathrm{I}_{1}$ above and beyond discrepancy diminishing by the cubic relation with Trial. Switching stimuli scale should perturb performance further without allowing any transfer of a veridical mastery of length.

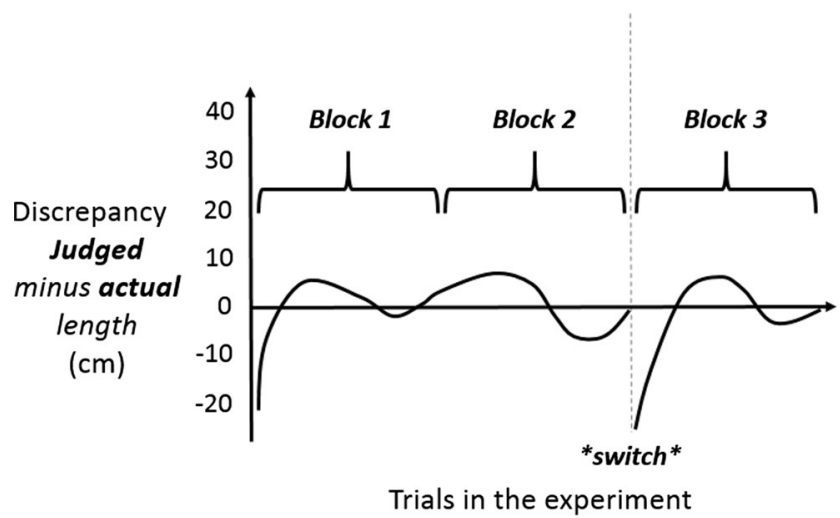

Fig. 1 Schematic of perceptual discrepancy depicting cubic trajectory across trials in blocks

\section{Method}

\section{Participants}

Fifteen undergraduate students aged 18-22 years (six females, nine males) participated after providing informed consent in accordance with Grinnell College's Institutional Review Board.

\section{Materials}

Stimulus objects were 40 roughly homogenous wooden dowels, $1.5 \mathrm{~cm}$ in diameter and of varying lengths (Table 1). The Short set comprised the first 20 dowels, the Long set comprised dowels number 21-40, and the Interleaved set comprised every other dowel from number 1 to 39 and then, in Block 3, every other dowel from 2 to 40. A string-andpulley system fixed to a table allowed for registering length judgments. To the right, a solid black curtain with an access slit covered the wielding area from floor to ceiling. The striking surface behind it was a $60.7 \times 60.7 \mathrm{~cm}$ square of $1-\mathrm{cm}$ thick cork mounted on 1-cm-thick plywood. ${ }^{3}$ The experimenters mounted the striking surface on the back of a chair that experimenters could pick up easily and move away during the free-wielding or place by the end of the stimulus object during striking. Experimenters used a black marker to draw a mark around the diameter of the dowel $9 \mathrm{~cm}$ from the edge demarcating a $9-\mathrm{cm}$ portion of the dowel serving as the handle.

\section{Procedure}

Behind the curtain, the experimenter handed dowels to participants randomly assigned to three experimenter conditions: Long-Short, Short-Long, and Interleaved. Participants sat before the pulley system, with their arm through the curtain. The experimenter placed the handle into the participant's open hand with black mark aligned with the inner edge of the palm between forefinger and thumb. The pulley system's marker always began from at zero-length position for each trial. Each trial included two length judgments for the same object: the first following wielding only and the second following

\footnotetext{
${ }^{3}$ Experimenters intended the cork mat to dampen some of the sound of impact. Acoustic energy resulting from the striking of a pendular hammer against an unseen object or the striking of a falling object against the floor can inform judgments of the unseen object's geometric properties (Carello, Anderson, \& Kunkler, 1998; Kunkler-Peck, \& Turvey, 2000; Wagman, Carello, Schmidt, \& Turvey, 2009). Pilot experiments contrived to decompose haptic from acoustic stimulation, having participants judge length following striking with or without headphones playing white noise found effects of object length and surface distance but, in comparison, no effect of the added acoustic information incident to striking (Carello, Fitzpatrick, \& Turvey, 1992).
} 
Table 1 Description of stimuli

\begin{tabular}{|c|c|c|}
\hline Length (cm) & Mass (g) & $\begin{array}{l}\text { First moment of inertia } \\
I\left(\mathrm{~g}^{*} \mathrm{~cm}^{2}\right)\end{array}$ \\
\hline 10 & 9 & 301.27 \\
\hline 12.5 & 15 & 783.36 \\
\hline 15 & 18 & 1352.53 \\
\hline 17.5 & 16 & 1635.58 \\
\hline 20 & 19 & 2536.01 \\
\hline 22.5 & 25 & 4222.27 \\
\hline 25 & 28 & 5837.27 \\
\hline 27.5 & 29 & 7314.50 \\
\hline 30 & 25 & 7503.52 \\
\hline 32.5 & 31 & $10,918.94$ \\
\hline 35 & 36 & $14,705.06$ \\
\hline 37.5 & 26 & $12,191.16$ \\
\hline 40 & 46 & $24,539.80$ \\
\hline 42.5 & 40 & $24,088.96$ \\
\hline 45 & 50 & $33,757.03$ \\
\hline 47.5 & 51 & $38,363.42$ \\
\hline 50 & 52 & $43,340.65$ \\
\hline 52.5 & 58 & $53,295.66$ \\
\hline 55 & 46 & $46,389.80$ \\
\hline 57.5 & 54 & $59,520.09$ \\
\hline 60 & 66 & $79,209.28$ \\
\hline 62.5 & 63 & $82,040.11$ \\
\hline 65 & 60 & $84,508.44$ \\
\hline 67.5 & 75 & $113,916.80$ \\
\hline 70 & 75 & $122,510.50$ \\
\hline 72.5 & 69 & $120,903.45$ \\
\hline 75 & 67 & $125,634.42$ \\
\hline 77.5 & 97 & $194,215.72$ \\
\hline 80 & 78 & $166,410.97$ \\
\hline 82.5 & 88 & $199,662.38$ \\
\hline 85 & 90 & $216,762.66$ \\
\hline 87.5 & 89 & $227,147.93$ \\
\hline 90 & 95 & $256,513.36$ \\
\hline 92.5 & 67 & $191,099.01$ \\
\hline 95 & 80 & $240,677.92$ \\
\hline 97.5 & 74 & $234,497.91$ \\
\hline 100 & 75 & $250,010.55$ \\
\hline 102.5 & 78 & $273,173.47$ \\
\hline 105 & 88 & $323,412.38$ \\
\hline 107.5 & 88 & $338,995.71$ \\
\hline
\end{tabular}

striking. For the first judgment, the experimenter instructed participants to wield the object freely and to register a judgment of its length on the pulley system, using the distance of the marker from the near end of the table. Wielding freely meant that the participant could wield in all directions, and the experimenter ensured that the striking surface was out of range of the stimulus object, preventing any accidental striking. Following the first judgment on each trial, the experimenter instructed the participants to leave the marker where they had placed it on the pulley system and to strike the unseen surface. Participants were allowed to strike until they made a second length judgment (Fig. 2; top panel). They struck so as to bring the distal tip of the dowel against the face of the panel, and the experimenter brought the panel back into range of the dowel and positioned it by the end of the dowel so that the participants needed only to swing the dowel to the right in the horizontal plane parallel to the floor. After the second judgment within a trial, the experimenter returned the marker to the zero-length position on the pulley system and again moved the striking panel out of range of the next dowel.

Previous research had found that the direction (i.e., left or right) of horizontal striking movements did not change participants' judgments (Stephen \& Arzamarski, 2009), and so the experimenters kept only horizontal movements to the right for striking in this study. Use of freely unconstrained wielding also replicated the method in from Stephen and Arzamarski (2009) as well as the general convention in dynamic-touch research to leave wielding behaviors wholly unconstrained (Arzamarski, Isenhower, Kay, Turvey, \& Michaels, 2010). This difference presents a possible confound, but attempts to make free wielding and striking more comparable would also risk introducing new information beyond experimental control. For instance, experimenters might follow the wielding behavior around with the striking surface so as to provide comparable mechanical information while also preserving freedom of wielding, but that method might contaminate participant's striking with any changes in the experimenter's contributions to the dowel-surface collisions.

Participants experienced each of 20 stimulus objects per set over the course of 20 trials. For the first two blocks, participants went through one set twice over. For the third block of 20 trials, the experimenter switched the stimulus objects without warning the participant: the Long-Short and the Short-Long groups received the shorter 20 and the longer 20 stimulus objects, respectively; and the Interleaved group received those objects with whole-numbercentimeter lengths for the first two blocks and those objects with .5-number-centimeter lengths for Block 3 (Fig. 2; bottom panel).

The experimenter noted any accidental striking of the object during wielding before the first judgment.

\section{Analysis}

Linear mixed-effect modeling tested effects of striking, switching, and group membership on the use of $\log \mathrm{I}_{1}$ from the stimulus objects, above and beyond any Block and Trial effects on the use of $\log \mathrm{I}_{1}$. A long-standing tradition in 


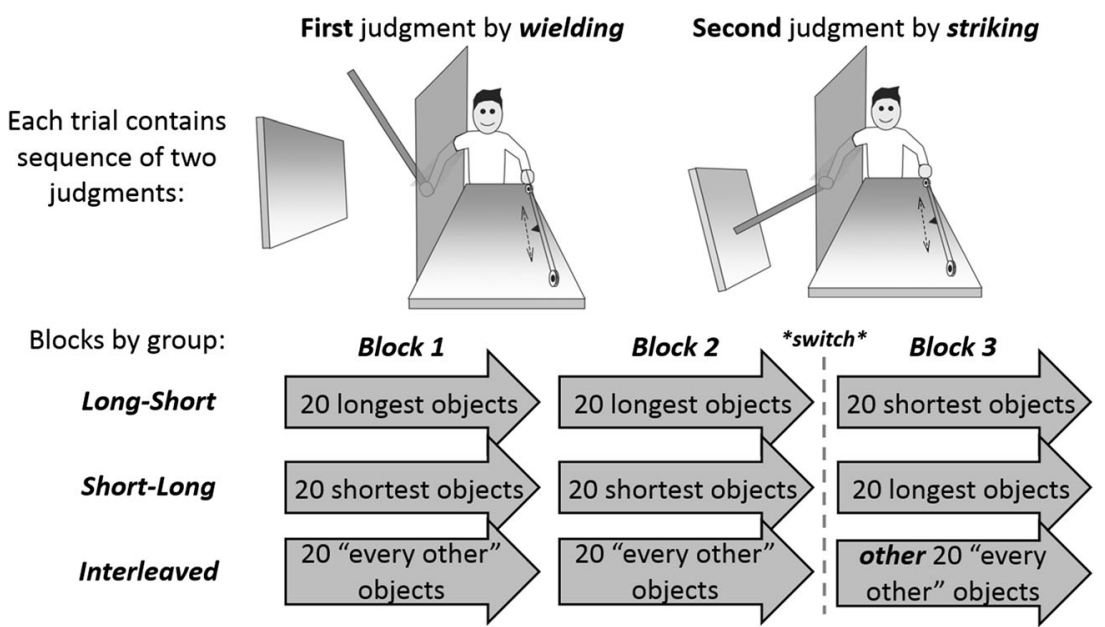

Fig. 2 Top panels schematize a participant making the first judgment per trial with only wielding (left) and the second judgment per trial with striking (right). Bottom panels schematize the progress by blocks of

dynamic touch literature is modeling linear relationships between informational variables and length judgments by considering intercepts and slopes of individual ordinary least-squares (OLS) regression models per participant (Withagen \& Michaels, 2005), intercepts addressing calibration (i.e., to the proper metric; e.g., definite scaling, Turvey \& Carello, 1995) and slopes addressing attunement (e.g., relative scaling). Mixed-effect modeling allows the hierarchical fitting of multiple participants' individual variable-to-judgment relationships all within one single statistical model using maximum likelihood (ML) instead of OLS, avoiding the need to estimate a new OLS model for each participant. Group membership, striking, and switching provided intercept effects, but trial, block, and $\log I_{1}$ served as slope effects. Intercept effects reflected what happened or what did not: participants were or were not in one or another group, participants struck leading up to a judgment or did not, and experimenters did (Block 3 ) or did not (Block 2) switch stimulus objects. Slope effects addressed the linear relationship between discrepancy and variables whose values fell along a continuous scale: for instance, trials increased from 1 to 20 , blocks increased from 1 to 3 , and $\log I_{1}$ increased across the values shown in Table 1.

Predictors Strike and Switch equaled 0 or 1 for first judgment or second, post-strike judgment and for Blocks 1 and 2 or Block 3, respectively. Predictor LS encoded condition equaling 1 for the Long-Short group, -1 for the Short-Long group, and 0 for the Interleaved group. We tested these effects first with linear effects of Trial and randomeffect intercept for individual participants (Model 1). We next tested these effects with orthogonal polynomials to cubic order of Trial and controlling for accidental striking, as well as random effects on Trial and $\log \mathrm{I}_{1}$ accounting for participants through the experiment in one of three groups (Long-Short, Short-Long, and Interleaved), and especially the switch between stimulus sets between Blocks 2 and 3

individual differences in the linear relationship between trial number and $\log \mathrm{I}_{1}\left(\right.$ Model 2). ${ }^{4}$

The random effects fit individual coefficients for each participant's performance within block so as to prevent individualparticipant differences from contaminating the estimation of fixed, sample-general effects. The absence of random effects besides an intercept for individual participants made Model 1 resemble more closely the standard repeated-measures ANOVA including fixed effects and the random effect of subject (Singer \& Willett, 2003). Model 2 includes random effects on Trial and $\operatorname{LogI}_{1}$ to absorb individual differences slopes effects beyond sample-general description (Withagen, \& van Wermeskerken, 2009). We included both models for two reasons: (1) to show that similar effects held irrespective of whether or not individual differences were explicitly modeled, and (2) to provide a simpler model (Model 1) that might helpfully lead towards understanding that allowing the mixed-effect model to address individual-participant differences in changes with Trial and $\log I_{1}$ would reveal the effect, across blocks in the task, of switching from one stimulus subset to the other (Model 2).

\section{Results}

Striking and practice diminished overestimation and reversed negative relationship between discrepancy and $\log I_{1}$.

\footnotetext{
${ }^{4}$ Conventional wisdom suggests that a ratio of $10-13$ observations per predictor is the minimum boundary to prevent overfitting (Babylak, 2004). Because we have two judgments on each of 60 trials from 15 participants, then participants completed 1800 separate judgments. Model 2 contains 26 fixed effects, 3 random effects, and also the 3 pairwise correlations among the random effects. In total, we thus have an observation-to-predictor ratio of $1800 / 32=56$, well above what is needed to prevent overfitting.
} 
Figure 3 depicts the model predictions from fixed effects in Model 1 (Table 2). Striking served to reduce judgments on each trial $(B=-7.20, S E=3.20, P<.05)$. Judgments diminished further over Block as well as over Trial within blocks $(B \mathrm{~s}=-14.15$ and $-1.45, S E \mathrm{~s}=5.33$ and .71 , both $P_{\mathrm{s}}<.05$, respectively). As found before (Stephen \& Arzamarski, 2009), discrepancy became more negative with greater $\log _{1}(B=-3.91, S E=1.03$, $P<.001)$, but this negative relationship diminished with Block and with Trial $(B \mathrm{~s}=1.57$ and $.17, S E \mathrm{~s}=.44$ and $.07, P_{\mathrm{S}}<.001$ and .05 ). Also as found before (Stephen \& Arzamarski, 2009), striking increased judgments of stimulus objects with greater $\operatorname{LogI}_{1}\left(\right.$ Strike $\times \operatorname{LogI}_{1} ; B=.79$, $S E=.30, P<.01)$.

Switching stimulus scales between Block 2 and Block 3 reinstated overestimation and negative relationship between discrepancy and $\log \mathrm{I}_{1}$. According to Model 1, switching from long to short or from short to long stimulus objects between the second and third block dramatically increased discrepancy between judged and actual length $(B=48.88, S E=13.31, P<.001)$. Switching also accentuated the negative relationship between discrepancy and $\operatorname{LogI}_{1}$ that might otherwise have diminished across blocks and trials $(B=-4.51, S E=1.06$, $P<.0001)$. However, there were no significant interactions of striking with switching stimulus objects, suggesting
Table 2 Basic regression model (Model 1) of discrepancies between judged length and actual length

\begin{tabular}{llll}
\hline Predictor & $B$ & $S E$ & $p$ \\
\hline Intercept & 45.51 & 12.15 & $<.001$ \\
Strike & -7.08 & 3.20 & $<.05$ \\
$\operatorname{LogI}_{1}$ & -3.91 & 1.03 & $<.001$ \\
LS & -9.89 & 5.31 & .07 \\
Switch & 48.88 & 13.31 & $<.001$ \\
Block & -14.15 & 5.33 & $<.05$ \\
Trial & -1.45 & .71 & $<.05$ \\
Strike $\times$ LogI 1 & .79 & .30 & $<.01$ \\
LS $^{\mathrm{a}} \times$ Switch & -6.64 & 13.18 & .62 \\
$\operatorname{LogI} I_{1} \times$ LS & .56 & .43 & .20 \\
$\operatorname{LogI}_{1} \times$ Switch & -4.51 & 1.06 & $<.0001$ \\
$\operatorname{LogI}_{1} \times$ Block & 1.57 & .44 & $<.001$ \\
$\operatorname{LogI}_{1} \times$ Trial & .17 & .07 & $<.05$ \\
Block $\times$ Trial & 1.01 & .33 & $<.01$ \\
$\operatorname{LogI}_{1} \times$ LS $\times$ Switch & 1.12 & 1.07 & .30 \\
$\operatorname{LogI}_{1} \times$ Block $\times$ Trial & -.11 & .03 & $<.001$ \\
\hline
\end{tabular}

a LS denotes the LongShort coding: 1 for the LongShort condition, -1 for the ShortLong condition, and 0 for the Interleaved condition

that striking continued to increase judgments with greater $\log I_{1}$.
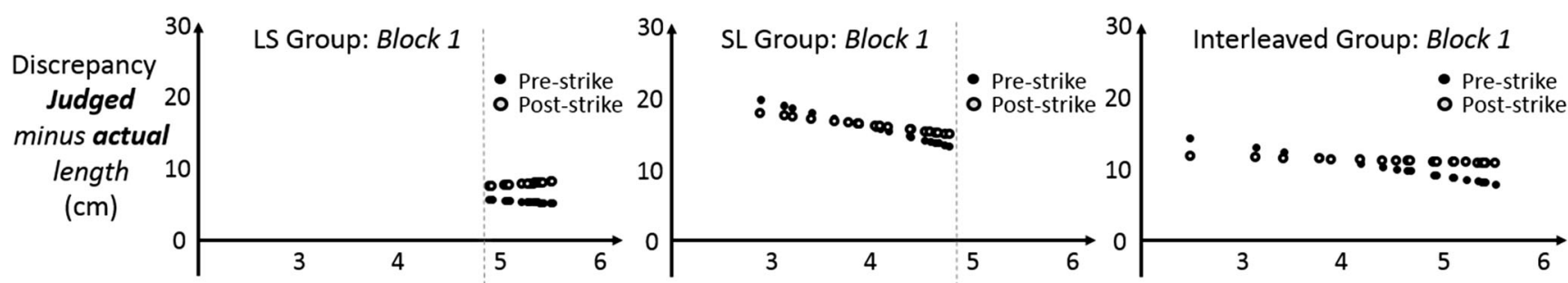

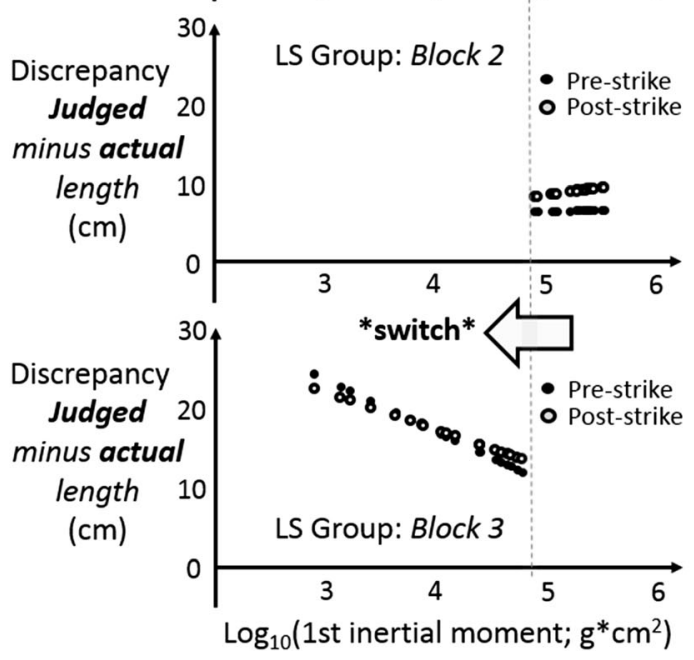

Fig. 3 Model predictions for perceptual discrepancy (judged length minus actual length) from fixed-effects from Model 1 (Table 2). Vertically, each row of panels corresponds to Block 1 (top), Block 2 (middle), and Block 3 (bottom). Horizontally, each column of panels corresponds to the

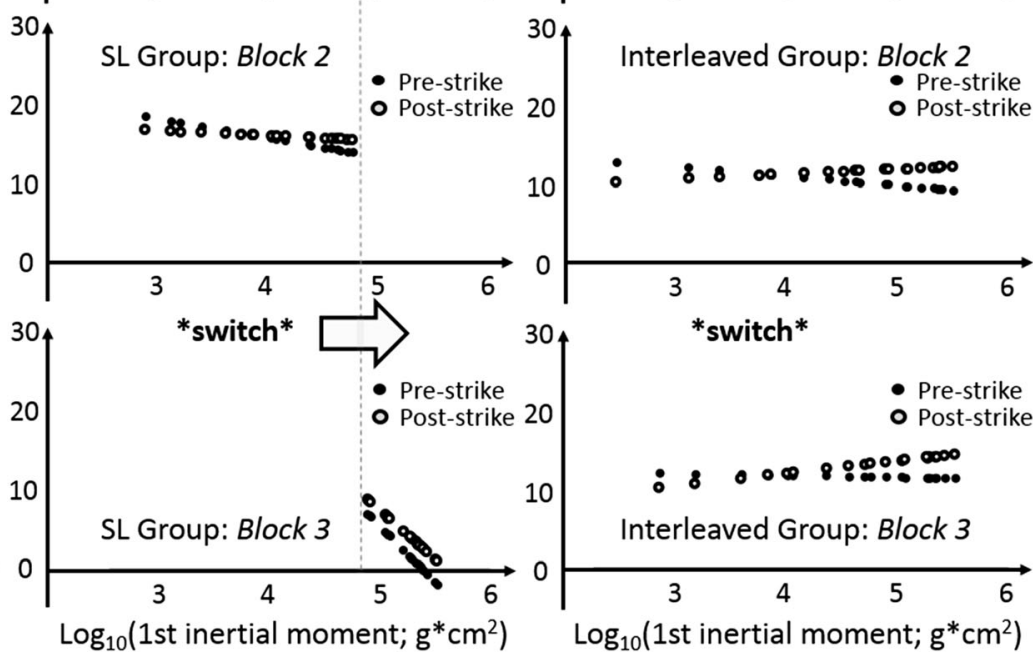

Long-Short (left), Short-Long (middle), and Interleaved (right) groups. Each panel plots predicted discrepancy against the logarithm of the first moment of inertia $\left(\operatorname{LogI}_{1}\right)$ 
Model 2: Incorporating cubic Trial terms, random-effect terms, and accidental striking terms reveals effect of switching stimuli scales.

Table 3 shows that the discrepancies do indeed reflect the cubic effects that we anticipated, showing significant effects of the linear and cubic effects of Trial. It is important to note both that the quadratic term was included but never significant and that the linear, quadratic, and cubic terms were all entered as orthogonal polynomials. Model 2 also included random effects for Trial (Linear) and $\log I_{1}$ to control for individual participant differences each of which significantly improved model fit, $\chi^{2}(2)=27.92, P<.0001$, and $\chi^{2}(3)=217.50$, $P<.0001$, respectively. Including accidental-strike terms revealed that accidental striking led to dramatic reductions in discrepancy between judged and actual length $(B=-65.31$, $S E=21.27, P<.01)$ but also that accidental striking increased judgments for objects with greater $\log \mathrm{I}_{1}(\mathrm{~B}=5.99, S E=1.84$, $P<.01)$. The intensification of the accidental striking effects may be due either to the participants' surprise upon feeling

Table 3 Enhanced regression model (Model 2) of discrepancies between judged length and actual length

\begin{tabular}{|c|c|c|c|}
\hline Predictor & $B$ & $S E$ & $p$ \\
\hline Intercept & 19.89 & 17.39 & .25 \\
\hline Strike & -7.17 & 2.86 & $<.05$ \\
\hline $\log _{1}$ & -1.32 & 1.82 & $<.001$ \\
\hline AccidentalStrike & -65.31 & 21.27 & $<.01$ \\
\hline $\mathrm{LS}^{\mathrm{a}}$ & -21.35 & 7.86 & $<.01$ \\
\hline Switch & 56.03 & 18.65 & $<.01$ \\
\hline Block & -1.96 & 8.70 & .82 \\
\hline Trial (Linear) & -612.77 & 198.52 & $<.01$ \\
\hline Trial (Quadratic) & 149.30 & 169.07 & .38 \\
\hline Trial (Cubic) & 357.22 & 174.07 & $<.05$ \\
\hline Strike $\times \operatorname{LogI}_{1}$ & .80 & .27 & $<.01$ \\
\hline $\mathrm{LS} \times$ Switch & 7.80 & 3.38 & $<.05$ \\
\hline $\operatorname{LogI}_{1} \times$ AccidentalStrike & 5.99 & 1.84 & $<.01$ \\
\hline $\operatorname{LogI}_{1} \times \operatorname{LS}$ & 1.43 & .79 & .07 \\
\hline $\operatorname{LogI}_{1} \times$ Switch & -5.27 & 1.91 & $<.01$ \\
\hline $\operatorname{LogI}_{1} \times$ Block & .32 & .93 & .73 \\
\hline $\operatorname{LogI}_{1} \times$ Trial (Linear) & 64.39 & 18.17 & $<.001$ \\
\hline $\operatorname{LogI}_{1} \times$ Trial (Quadratic) & -10.56 & 15.79 & .50 \\
\hline $\operatorname{LogI}_{1} \times$ Trial $($ Cubic $)$ & -30.35 & 16.13 & .06 \\
\hline Block $\times$ Trial $($ Linear $)$ & 260.28 & 93.62 & $<.01$ \\
\hline Block × Trial (Quadratic) & -83.76 & 77.47 & .28 \\
\hline Block $\times$ Trial $($ Cubic $)$ & -261.29 & 81.04 & $<.05$ \\
\hline $\log \mathrm{I}_{1} \times$ Block $\times$ Trial $($ Linear $)$ & -28.09 & 8.54 & $<.001$ \\
\hline $\operatorname{LogI}_{1} \times$ Block $\times$ Trial (Quadratic) & 7.08 & 7.24 & .33 \\
\hline $\log \mathrm{I}_{1} \times$ Block $\times$ Trial $($ Cubic $)$ & 22.68 & 7.49 & $<.01$ \\
\hline
\end{tabular}

${ }^{\text {a }}$ LS denotes the LongShort coding: 1 for the LongShort condition, -1 for the ShortLong condition, and 0 for the Interleaved condition unplanned contact with an unseen surface or to the relatively small sample of accidental strikings (38 total within 900 trials).

Otherwise, all effects that appeared in Model 1 bore comparable direction and significance in Model 2, but an important change was that the improvements of Model 2 above and beyond Model 1 now revealed a significant difference in the effect of switching stimuli based on experimental group. Whereas LS $\times$ Switch had appeared to be nonsignificant in Model 1, Model 2 indicates a significant positive effect for LS $\times$ Switch $(B=7.80, S E=3.38$, $P<.05$ ). Because of the coding of LS (i.e., 1 for LongShort group, -1 for Short-Long group, and 0 for Interleaved group), this positive effect indicates that the post-switch increase in discrepancy (already noted in Model 1 and persisting in Model 2; $B=56.03, S E=18.65, P<.01$ ) was significantly greater for the Long-Short group and significantly lesser for the Short-Long group.

Figures 4 through 6 show the discrepancies for each length judgment from actual length for participants in the Long-Short group, the Short-Long group, and the Interleaved group, respectively (actual discrepancies in black lines; model-predicted discrepancy in grey). We note that Figs. 4, 5 and 6 feature some extremely large, sudden increases or decreases in discrepancy (e.g., bottom-right panel of Fig. 4 and top-right panel of Fig. 6). These trials are evenly distributed across only-wielding and poststriking trials.

Interleaved condition showed strengthening correlation of judgments with length over blocks. Table 4 shows the signed discrepancy, absolute discrepancy, and correlation coefficient averaged for each block in each condition. Discrepancy remains relatively constant and positive in the Interleaved condition, indicating a moderate case of the overestimation exhibited in the other two conditions, but unlike in the Long-Short and Short-Long conditions, correlation coefficients increased across blocks for the Interleaved condition.

Mixed-effect modeling of length judgments with no random effects besides the individual-participant intercept estimated fixed effects for the interactions Length $\times$ LS $\times$ Switch and Length $\times$ Block and all component main effects and lower-order interactions. Long-Short and Short-Long participants underestimated and overestimated, respectively (LS; $B=-8.35, S E=2.90, P<.01)$, and switching in Block 3 contributed to overestimation (Switch; $B=16.50, S E=5.81$, $P<.01$ ). Length judgments had just below a slope of 1 with respect to actual length (Object length; $B=.92, S E=.04$, $P<.0001$ ), and relationships between length judgments and actual length for the Interleaved condition with each block in the task (Length $\times$ Block; $B=.04, S E=.02, P<.05$ ). As for the other two conditions, Long-Short and Short-Long participants showed an exaggerated and diminished, respectively, 


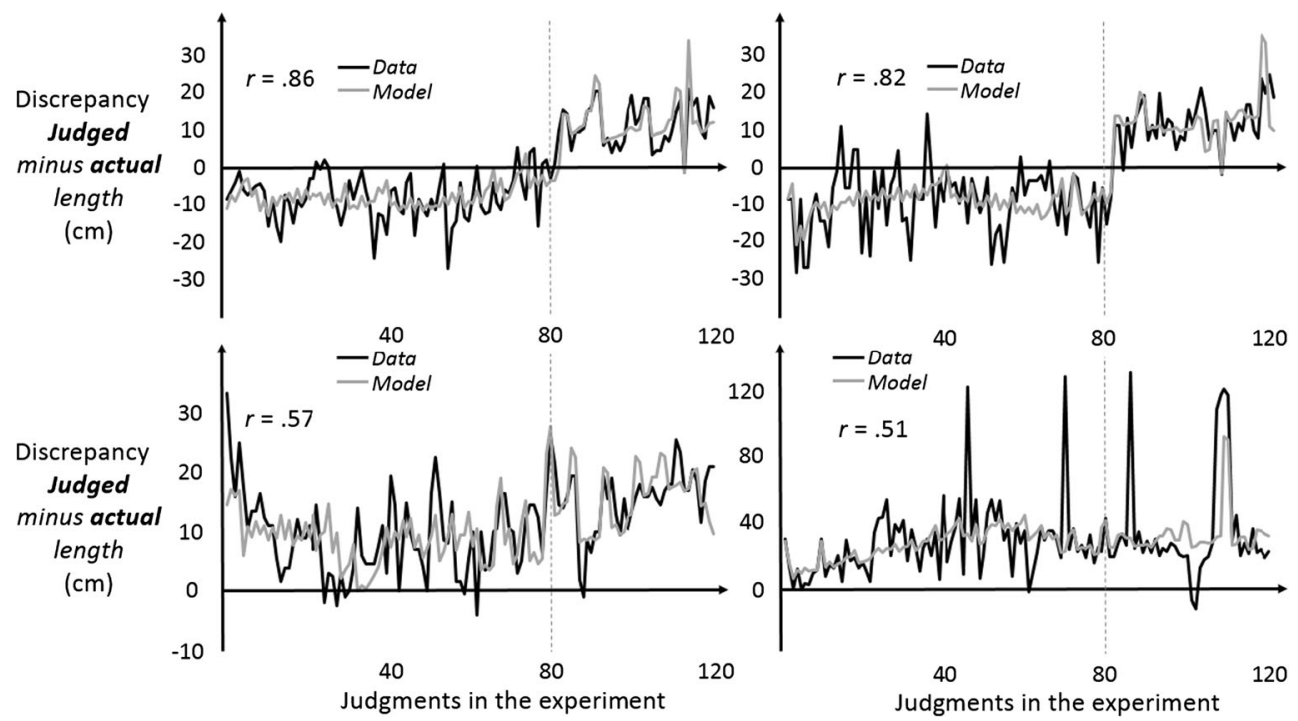

Fig. 4 Individual participants' perceptual-discrepancy data in LongShort group (black) compared to predicted perceptual discrepancy according to Model 2 (shown in Table 3; grey) for all judgments that they

effect of length on perceived length (Length $\times \mathrm{LS} ; B=.07$, $S E=.03, P<.01)$. Furthermore, the relationship of length judgments with actual length for participants in both of these conditions diminished in the third block (Length $\times$ Switch; $B=-.29, S E=.03, P<.0001)$. Hence, Long-Short and Short-Long conditions fail to exhibit both definite scaling and relative scaling (e.g., Turvey \& Carello, 1995). However, despite poor calibration to scale of the actual length, the Interleaved condition attuned better to mechanical variables specifying length than participants in the other conditions did (e.g., Jacobs \& Michaels, 2007; Withagen, \& Caljouw, 2011). produced. We chose to depict participant data with the two best and two worst correlation coefficients $r$ between data and model

\section{Discussion}

This study revisited self-training dynamic touch by striking with two crucial design changes: (1) allowing participants to strike on each trial and correct judgments accordingly, and (2) allowing participants to train themselves using striking with one stimulus scale over the first two blocks and, without warning them explicitly, to change the stimulus scale for the third block. If self-training promoted veridical judgments of length, then repeated striking should have stabilized length judgments at zero-discrepancy, and the self-training should have led to transfer of relatively stable zero-discrepancy from one

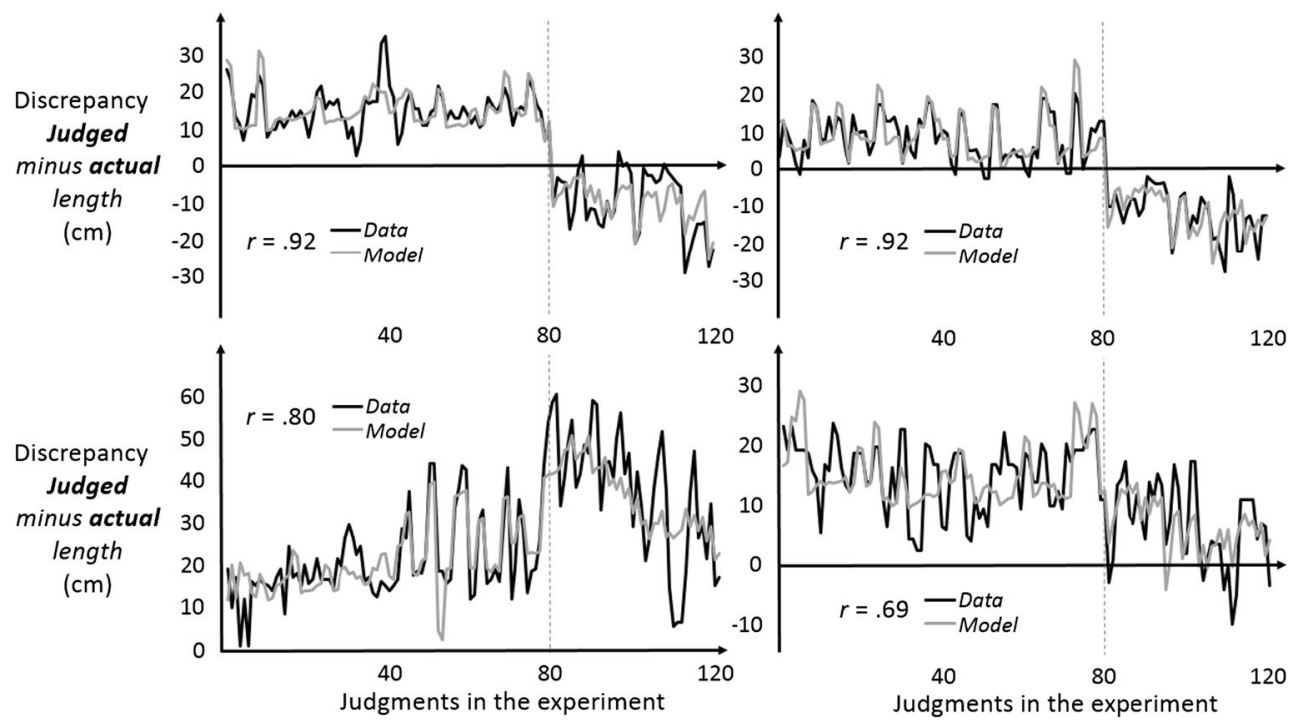

Fig. 5 Individual participants' perceptual-discrepancy data in ShortLong group (black) compared to predicted perceptual discrepancy according to Model 2 (shown in Table 3; grey) for all judgments that they produced. We chose to depict participant data with the two best and two worst correlation coefficients $r$ between data and model 


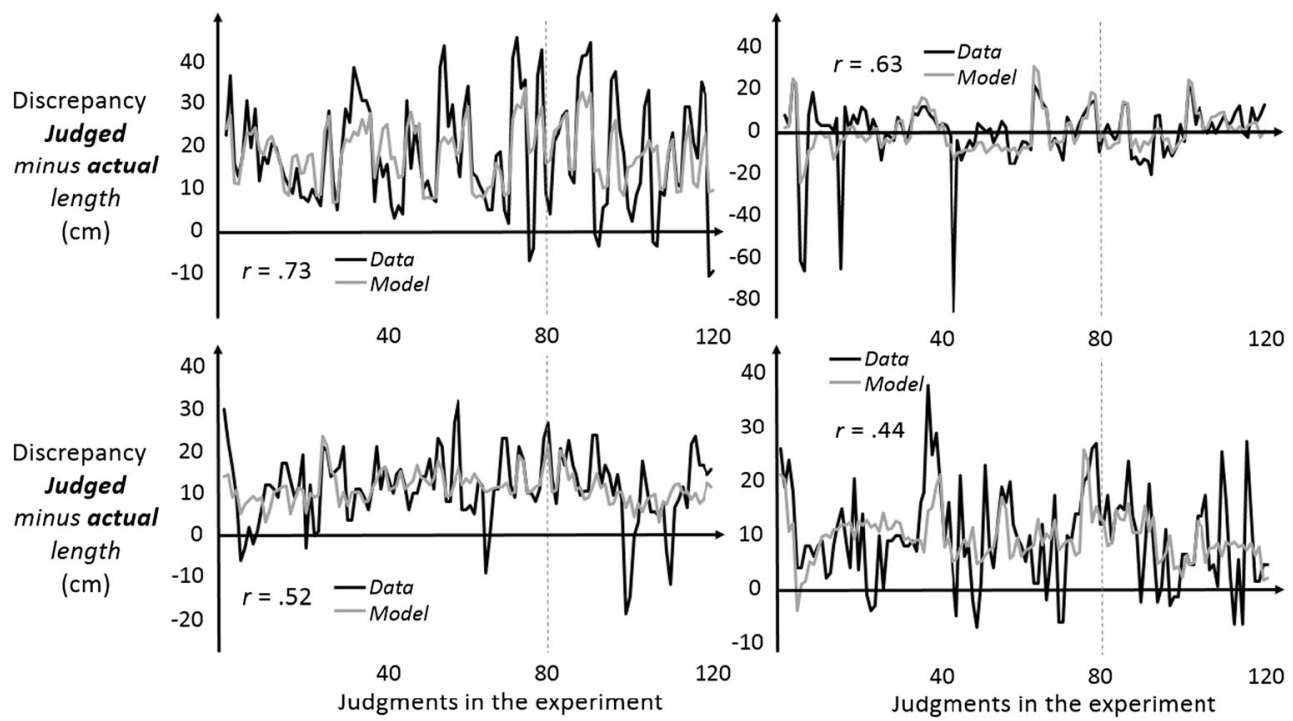

Fig. 6 Individual participants' perceptual-discrepancy data in Interleaved group (black) compared to predicted perceptual discrepancy according to Model 2 (shown in Table 3; grey) for all judgments that they

stimulus subset to another. Results contradicted these expectations and were more consistent with the alternate possibility that striking serves rather to exaggerate length for objects with greater $\log _{1}$. The Interleaved condition allowed participants to better attune their judgments to actual length, but this better attunement did not undermine the generally exaggerating effect of striking on length judgments, suggesting that repeated experience striking the same stimulus subset improved relative scaling but not definite scaling. Strangely, participantgenerated haptic experiences with wielded objects might make haptic perception less veridical.

The possibility of building response patterns solely through self-generated interactions with context and without stipulated instruction aligns well with a theoretical concept called selforganization (Barsalou, Breazeal, \& Smith, 2007; Kelso, 1995; Soberano \& Kelty-Stephen, 2015; Thelen \& Smith, 1994). Self-organization provides a framework for understanding haptic perception of objects through self-training because produced. We chose to depict participant data with the two best and two worst correlation coefficients $r$ between data and model

participants are bootstrapping their own changes in response through their own mechanical interactions with context and without the benefit of an experimenter's stipulated, explicit feedback to instruct them (Abney, Wagman, \& Schneider, 2014). The present results indicate that self-organization need not generate veridical responses. Self-organization may be an important framework in which to consider learning in general (Kelty-Stephen \& Dixon, 2014; Spencer, Thomas, \& McClelland, 2009; Thelen \& Smith, 1994), but we should not forget that some of the most pioneering and programmatic work in applying self-organization to psychology dealt with a perceptual discrepancy, namely, the Piagetian A-not-B error in which the developing infant's mind systematically misaligns haptic coordination of infant reaching with visual information about target location, leading to a systematically non-zero discrepancy between reach and target (e.g., Clearfield, Dineva, Smith, Diedrich, \& Thelen, 2009; Spencer, Dineva, \& Smith, 2009; Spencer, Perone, \& Buss, 2011).

Table 4 Means and standard errors for signed discrepancies, absolute discrepancies, and Pearson correlation coefficients between perceived length and actual length, by block and by condition

\begin{tabular}{lllll}
\hline Condition & Measure & Block 1 & Block 2 & Block 3 \\
\hline LongShort & Signed discrepancy & $6.57(6.60)$ & $8.53(7.86)$ & $18.03(4.11)$ \\
& Absolute discrepancy & $13.54(2.91)$ & $15.17(4.74)$ & $18.03(4.11)$ \\
& Correlation coefficient $r$ & $.83(.04)$ & $.82(.08)$ & $.78(.16)$ \\
ShortLong & Signed discrepancy & $14.71(1.94)$ & $17.03(3.19)$ & $19.80(9.42)$ \\
& Absolute discrepancy & $14.71(1.94)$ & $17.03(3.19)$ & $.95(.01)$ \\
Interleaved & Correlation coefficient $r$ & $.94(.01)$ & $11.02(3.58)$ & $16.46(5.43)$ \\
& Signed discrepancy & $10.16(2.89)$ & $11.86(2.83)$ & $.05)$ \\
& Absolute discrepancy & $10.16(2.89)$ & $.94(.03)$ & $.95(.01)$ \\
\hline
\end{tabular}




\section{References}

Abney, D. H., Wagman, J. B., \& Schneider, J. (2014). Changing grasp position on a wielded object provides self-training for perception of length. Attention, Perception \& Psychophysics, 76, 247-254.

Arzamarski, R., Isenhower, R. W., Kay, B. A., Turvey, M. T., \& Michaels, C. F. (2010). Effects of intention and learning on attention to information in dynamic touch. Attention, Perception \& Psychophysics, $72,721-735$.

Babylak, M. A. (2004). What you see may not be what you get: A brief, nontechnical introduction to overfitting in regression-type models. Psychosomatic Medicine, 66, 411-421.

Barsalou, L. W., Breazeal, C., \& Smith, L. B. (2007). Cognition as coordinated non-cognition. Cognitive Processing, 8, 79-91.

Carello, C., Fitzpatrick, P., \& Turvey, M. T. (1992). Haptic probing: Perceiving the length of a probe and the distance of a surface probed. Perception \& Psychophysics, 51, 580-598.

Carello, C., Anderson, K. L., \& Peck, A. (1998). Perception of object length by sound. Psychological Science, 9, 211-214.

Clearfield, M. W., Dineva, E., Smith, L. B., Diedrich, F. L., \& Thelen, E. (2009). Cue salience and infant perseverative reaching: Tests of dynamic field theory. Developmental Science, 12, 26-40.

Fitzpatrick, P., Carello, C., \& Turvey, M. T. (1994). Eigenvalues of the inertia tensor and exteroception by the "muscular sense.". Neuroscience, 60, 551-568.

Jacobs, D. M., \& Michaels, C. F. (2007). Direct learning. Ecological Psychology, 19, 321-349.

Kelso, J. A. S. (1995). Dynamic patterns. Cambridge: MIT Press.

Kelty-Stephen, D. G., \& Dixon, J. A. (2014). Interwoven fluctuations during intermodal perception: Fractality in head sway supports the use of visual feedback in haptic perceptual judgments by manual wielding. Journal of Experimental Psychology: Human Perception \& Performance, 40, 2289-2309.

Kunkler-Peck, A. J., \& Turvey, M. T. (2000). Hearing shape. Journal of Experimental Psychology: Human Perception \& Performance, 26, 279-294.

Singer, J. D., \& Willet, J. B. (2003). Applied longitudinal data analysis. New York: Oxford University Press.
Soberano, E. K., \& Kelty-Stephen, D. G. (2015). Demystifying cognitive science: Explaining cognitive science through network-based modeling. Frontiers in Physiology, 6, 88 .

Spencer, J. P., Dineva, E., \& Smith, L. B. (2009). Comment on "Infants' perseverative search errors are induced by pragmatic misinterpretation. Science, 325, 1625-a.

Spencer, J. P., Thomas, M. S. C., \& McClelland, J. L. (Eds.). (2009). Toward a unified theory of development: Connectionism and $d y$ namic systems theory re-considered. New York: Oxford University Press.

Spencer, J. P., Perone, S., \& Buss, A. T. (2011). Twenty years and going strong: A dynamic systems revolution in motor and cognitive development. Child Development Perspectives, 5, 260-266.

Stephen, D. G., \& Arzamarski, R. (2009). Self-training of dynamic touch: Striking improves judgment by wielding. Attention, Perception \& Psychophysics, 71, 1717-1723.

Thelen, E., \& Smith, L. B. (1994). A dynamic-systems approach to the development of cognition and action. Cambridge: MIT Press.

Turvey, M. T., \& Carello, C. (1995). Dynamic touch. In W. Epstein \& S. Rogers (Eds.), Handbook of perception and cognition (Perception of space and motion, Vol. V, pp. 401-490). San Diego: Academic.

van de Langenberg, R., Kingma, I., \& Beek, P. J. (2008). The perception of limb orientation depends on the center of mass. Journal of Experimental Psychology: Human Perception \& Performance, 34, 624-639.

Wagman, J. B., Carello, C., Schmidt, R. C., \& Turvey, M. T. (2009). Is perceptual learning unimodal? Ecological Psychology, 21, 37-67.

Withagen, R., \& Caljouw, S. R. (2011). Aging effects attunement in perceiving length by dynamic touch. Attention, Perception, \& Psychophysics, 73, 1216-1226.

Withagen, R., \& Michaels, C. F. (2005). The role of feedback information for calibration and attunement in perceiving length by dynamic touch. Journal of Experimental Psychology: Human Perception \& Performances, 31, 1379-1390.

Withagen, R., \& van Wermeskerken, M. (2009). Individual differences in learning to perceive length by dynamic touch: Evidence for variation in individual learning capacities. Attention, Perception \& Psychophysics, 71, 64-75. 